\title{
Feng's First Integral Method Applied to the ZKBBM and the Generalized Fisher Space-Time Fractional Equations
}

\author{
Huitzilin Yépez-Martínez, Ivan O. Sosa, and Juan M. Reyes \\ Universidad Autónoma de la Ciudad de México, Prolongación San Isidro 151, Colonia San Lorenzo Tezonco, \\ Delegación Iztapalapa, 09790 México DF, Mexico
}

Correspondence should be addressed to Huitzilin Yépez-Martínez; huitzilin.yepez.martinez@uacm.edu.mx

Received 22 November 2014; Revised 13 January 2015; Accepted 14 January 2015

Academic Editor: Claudio Cuevas

Copyright (C) 2015 Huitzilin Yépez-Martínez et al. This is an open access article distributed under the Creative Commons Attribution License, which permits unrestricted use, distribution, and reproduction in any medium, provided the original work is properly cited.

\begin{abstract}
The fractional derivatives in the sense of the modified Riemann-Liouville derivative and Feng's first integral method are employed to obtain the exact solutions of the nonlinear space-time fractional ZKBBM equation and the nonlinear space-time fractional generalized Fisher equation. The power of this manageable method is presented by applying it to the above equations. Our approach provides first integrals in polynomial form with high accuracy. Exact analytical solutions are obtained through establishing first integrals. The present method is efficient and reliable, and it can be used as an alternative to establish new solutions of different types of fractional differential equations applied in mathematical physics.
\end{abstract}

\section{Introduction}

Fractional differential equations have been the focus of many studies due to their frequent appearance in various applications in physics, biology, engineering, signal processing, systems identification, control theory, finance, and fractional dynamics [1-3]. Recently, a large amount of literature has been provided to construct the solutions of fractional ordinary differential equations, fractional partial differential equations (PDEs), and integral equations of physical interest. Several powerful methods have been proposed to obtain approximate and exact solutions of fractional differential equations, such as the Adomian decomposition method [4, 5], the variational iteration method [6-8], the homotopy analysis method [9-12], the homotopy perturbation method [13-15], the Lagrange characteristic method [16], and the fractional subequation method [17].

In [18], Jumarie proposed a modified Riemann-Liouville derivative. With this kind of fractional derivative and some useful formulas, we can convert fractional differential equations into integer-order differential equations by variable transformation.

Feng [19] has introduced a reliable and effective method called Feng's first integral method to look for travelling wave solutions of nonlinear PDEs. The basic idea of this method is to construct a first integral with polynomial coefficients of an explicit form to an equivalent autonomous planar system by using division theorem. This method in comparison with other methods has many advantages; it avoids a great deal of complicated and tedious calculation and provides exact and explicit travelling solutions with high accuracy.

Feng's first integral method [20-26] can be used to construct the exact solutions for some time fractional differential equations.

Among the nonlinear PDEs there are two important examples of fundamental interest in mathematical-physical models: the nonlinear ZKBBM equation that describes the wave water phenomena in the large wavelength limit and one special case of nonlinear reaction-diffusion equation better known as the generalized Fisher equation. Let us review some general aspects about these two types of nonlinear PDEs.

The well-known Zakharov-Kuznetsov-Benjamin-BonaMahony equation (ZKBBM equation) also referred to as the regularized long wave equation (see $[27,28]$ )

$$
\begin{aligned}
U_{t}(x, t)+U_{x}(x, t)-2 a U(x, t) U_{x}(x, t)-b U_{t x x}(x, t) & =0, \\
t & >0,
\end{aligned}
$$


was originally derived for water waves under the assumption of small wave amplitude and large wavelength. The ZKBBM equation describes approximately the unidirectional propagation of long waves in certain nonlinear dispersive systems and has been introduced in order to give a satisfactory description for the small wave amplitude $U(x, t)$ in the large wavelength regime in many other physical systems. It has been used to account adequately for observable phenomena such as the interaction of solitary waves and dissipationless undular shocks; see [27].

The details of the derivation of the ZKBBM equation differ, in different examples, but in general the essentials are as follows. The first property of the systems in question is that dispersive effects on infinitesimal waves vanish in the limit as wavelength becomes infinite, and the limiting phase speed is a constant $\left(c_{0}>0\right)$. Thus waves of extreme length, along the $+x$ propagation direction, are described by

$$
U_{t}(x, t)+c_{0} U_{x}(x, t)=0, \quad t>0,
$$

whose general solution is an arbitrary differentiable function of $x-c_{0} t$, where $U_{t}=\partial U / \partial t$ and $U_{x}=\partial U / \partial x$. In certain applications (2) already has some validity as an approximation for real waves of sufficiently small amplitude and large wavelength but is not a valid approximation for very large times, during which nonlinear and frequency dispersive effects are accumulated to a significant level. These effects require the introduction of at least a first order approximation model to extend the range of validity of the model (1).

For the linearized equation (1) all values of $U(x, t)$ are propagated with the same velocity $(d x / d t)=c_{0}$. When the nonlinear effects are taken into account, the characteristic velocity becomes dependent linearly on $U(x, t)$ for the waves of finite but small amplitude; therefore in the first order approximation we obtain

$$
\frac{1}{c_{0}}\left(\frac{d x}{d t}\right)_{u=\text { const. }}=1+\beta U(x, t),
$$

where $\beta$ is a constant. After the substitution of (3) into (2) the following equation is obtained:

$$
U_{t}(x, t)+c_{0} U_{x}(x, t)+c_{0} \beta U(x, t) U_{x}(x, t)=0, \quad t>0 .
$$

Additionally the dispersive effects as suffered by waves of finite but large wavelength in the model (2) are ignored. In the first order approximation the dispersive effects transform the model (2) into the following equation:

$$
U_{t}(x, t)+c_{0}\left(U(x, t)+\alpha U_{t x}(x, t)\right)_{x}=0, \quad t>0 .
$$

Although for a self-consistent theory the two kinds of small effects have to be considered simultaneously, the outcome of such theories can generally be anticipated by considering the two effects separately. That is, in a first approximation, the above corrections are added separately into (2), and higher order terms appear negligible in this approximation.

When the physical parameters and the scaling factors presented in a particular example are appropriately absorbed into the definition of the dependent variable $U(x, t)$ and the independent variables $x$ and $t$, which are, respectively, proportional to distance in the physical system and to time, the ZKBBM equation is given by:

$$
\begin{gathered}
U_{t}(x, t)+U_{x}(x, t)-2 a U(x, t) U_{x}(x, t) \\
-b U_{t x x}(x, t)=0, \quad t>0 .
\end{gathered}
$$

Equation (6) can be extended in order to consider fractional order derivatives obtaining the ZKBBM space-time fractional equation [29]:

$$
\begin{aligned}
D_{t}^{\alpha} U(x, t)= & -D_{x}^{\alpha} U(x, t)+2 a U(x, t) D_{x}^{\alpha} U(x, t) \\
& +b D_{t}^{\alpha}\left(D_{x}^{2 \alpha} U(x, t)\right) \quad t>0,0<\alpha \leq 1,
\end{aligned}
$$

where $D_{x}^{\alpha} U(x, t)$ and $D_{t}^{\alpha} U(x, t)$ denotes the fractional derivative of order $\alpha$ with respect to the independent variables $x$ and $t$.

The second nonlinear PDE mentioned before, the generalized Fisher equation [30-32], can be obtained as a special case of the reaction-diffusion equation. The reactiondiffusion equation plays a fundamental role in a great number of various models of heat and reaction-diffusion processes, mathematical biology, chemistry, genetics, and many other fields.

We start with the reaction-diffusion equation with power nonlinearity (see, for example, [33]):

$$
U_{t}(x, t)-U_{x x}(x, t)=f(U(x, t)), \quad t>0 ;
$$

here $f(U(x, t))$ is a sufficiently smooth polynomial function satisfying the relations $f(0)=f(1)=0$.

One particular expression for the function $f(U(x, t))$ in (8) which satisfies these conditions is [33]

$$
\begin{aligned}
U_{t}(x, t)- & U_{x x}(x, t) \\
=k & \left(-(k+1) U^{n}(x, t)+\lambda_{1} U(x, t)+\lambda_{2} U^{(n+1) / 2}(x, t)\right. \\
& \left.+\lambda_{3} U^{(3-n) / 2}(x, t)+\lambda_{4} U^{2-n}(x, t)\right), \quad t>0,
\end{aligned}
$$

where $\lambda_{1}, \ldots, \lambda_{4}$ are constants that satisfy the condition imposed over $f(U(x, t))$ and $n$ is an arbitrary constant. Equation (9) defines a special class of the nonlinear heat equation, which includes many important applications in some of the areas mentioned before. If we consider the special case in (9) where $\lambda_{2}=\lambda_{3}=\lambda_{4}=0, n=6$, and $\lambda_{1}=k(k+1)=$ 1, we obtain the generalized Fisher equation:

$$
U_{t}(x, t)-U_{x x}(x, t)=U(x, t)\left(1-U^{6}(x, t)\right), \quad t>0,
$$

where in the original mathematical biology model $U(x, t)$ denotes the population density and $U(x, t)\left(1-U^{6}(x, t)\right)$ represents the population supply due to births and deaths.

The above equation has been introduced as a model for the propagation of a mutant gene in mathematical biology 
[30-32] and can be extended to fractional order derivatives obtaining the generalized Fisher space-time fractional equation:

$$
\begin{array}{r}
D_{t}^{\alpha} U(x, t)=D_{x}^{2 \alpha} U(x, t)+U(x, t)\left(1-U^{6}(x, t)\right), \\
t>0, \quad 0<\alpha \leq 1 .
\end{array}
$$

The present work investigates the applicability and effectiveness of Feng's first integral method to obtain new exact analytical solutions for the nonlinear space-time fractional Zakharov-Kuznetsov-Benjamin-Bona-Mahony (ZKBBM) partial differential equation and the nonlinear space-time fractional generalized Fisher equation.

We will show that, for the analytical solutions of the ZKBBM fractional partial differential equation, one of the advantages of Feng's first integral method is the evaluation of the constants involved in the analytical solutions that have not been evaluated within the subequation method [29]. Furthermore for the analytical solutions of the space-time fractional generalized Fisher equation, by applying Feng's first integral method, we will obtain three new analytical solutions that have not been obtained in previous works [30,31,33].

\section{The Modified Riemann-Liouville Derivative and Feng's First Integral Method}

In this section we present the main ideas of Feng's first integral method. This method considers the Jumarie modified Riemann-Liouville fractional derivative of order $\alpha$; we first give some definitions and properties of the modified Riemann-Liouville derivative which are used further in this paper.

Assume that $f: \mathbb{R} \rightarrow \mathbb{R}, x \rightarrow f(x)$ denotes a continuous (but not necessarily differentiable) function. The Jumarie modified Riemann-Liouville derivative of order $\alpha$ is defined by the expression [18]

$$
\begin{aligned}
& D_{x}^{\alpha} f(x) \\
& \quad=\left\{\begin{aligned}
\frac{1}{\Gamma(1-\alpha)} \int_{0}^{x}(x-\xi)^{-\alpha-1} & \\
\cdot[f(\xi)-f(0)] d \xi, & \alpha<0, \\
\frac{1}{\Gamma(1-\alpha)} \frac{d}{d x} \int_{0}^{x}(x-\xi)^{-\alpha} & \\
\cdot[f(\xi)-f(0)] d \xi & 0<\alpha<1, \\
{\left[f^{(\alpha-n)}(x)\right]^{(n)} } & n \leq \alpha \leq n+1, n \geq 1 .
\end{aligned}\right.
\end{aligned}
$$

Some properties of the fractional modified RiemannLiouville derivative are

$$
\begin{gathered}
D_{x}^{\alpha} x^{\gamma}=\frac{\Gamma(\gamma+1)}{\Gamma(\gamma+1-\alpha)} x^{\gamma-\alpha}, \\
D_{x}^{\alpha}(f(x) g(x))=g(x)\left(D_{x}^{\alpha} f(x)\right)+f(x)\left(D_{x}^{\alpha} g(x)\right),
\end{gathered}
$$

$$
\begin{aligned}
D_{x}^{\alpha} f[g(x)] & =f_{g}^{\prime}[g(x)] D_{x}^{\alpha} g(x) \\
& =\left(D_{g}^{\alpha} f[g(x)]\right)\left(g^{\prime}(x)\right)^{\alpha} .
\end{aligned}
$$

Now in order to introduce Feng's first integral method [19], let us consider the space-time fractional differential equation with independent variables $x_{1}, x_{2}, \ldots, x_{m}, t$ and some function $u$ :

$$
\begin{gathered}
F\left(u, D_{t}^{\alpha} u, D_{x_{1}}^{\alpha} u, D_{x_{2}}^{\alpha} u, D_{x_{3}}^{\alpha} u, \ldots, D_{t}^{2 \alpha} u,\right. \\
\left.D_{x_{1}}^{2 \alpha} u, D_{x_{2}}^{2 \alpha} u, D_{x_{3}}^{2 \alpha} u, \ldots\right)=0 .
\end{gathered}
$$

Using the variable transformation

$$
\begin{gathered}
u\left(x_{1}, x_{2}, \ldots, x_{m}, t\right)=U(\xi), \\
\xi=\frac{l_{1} x_{1}^{\alpha}+l_{2} x_{2}^{\alpha}+\cdots+l_{m} x_{m}^{\alpha}+\lambda t^{\alpha}}{\Gamma(1+\alpha)},
\end{gathered}
$$

where $l_{i}$ and $\lambda$ are constants to be determined later, the fractional differential equation (14) is reduced to a nonlinear ordinary differential equation

$$
H=H\left(U(\xi), U^{\prime}(\xi), U^{\prime \prime}(\xi), \ldots\right),
$$

where $U^{\prime}(\xi)=d U(\xi) / d \xi$.

We assume that (16) has a solution in the form

$$
U(\xi)=X(\xi),
$$

and we introduce a new independent variable $Y(\xi)=X^{\prime}(\xi)$, which leads to the following new system of equations:

$$
\begin{gathered}
X^{\prime}(\xi)=Y(\xi), \\
Y^{\prime}(\xi)=G(X(\xi), Y(\xi)) .
\end{gathered}
$$

Now, let us introduce the central idea of Feng's first integral method. By using the division theorem for two variables in the complex domain $\mathbb{C}$ which is based on the HilbertNullstellensatz theorem [34], we can obtain one first integral to (18) which can reduce (16) to a first order integrable ordinary differential equation. An exact solution to (14) is then obtained by solving this equation directly.

Division Theorem. Suppose that $P(x, y)$ and $Q(x, y)$ are polynomials in $\mathbb{C}[x, y]$, and $P(x, y)$ is irreducible in $\mathbb{C}[x, y]$. If $Q(x, y)$ vanishes at all zero points of $P(x, y)$, then there exists a polynomial $H(x, y)$ in $\mathbb{C}[x, y]$ such that

$$
Q(x, y)=P(x, y) H(x, y) .
$$

\section{Applications}

In this section, we illustrate the applicability of Feng's first integral method to solve the nonlinear fractional partial differential equations (7) and (11). 
Example 1. As a first example we consider the space-time fractional ZKBBM equation (7):

$$
\begin{aligned}
D_{t}^{\alpha} U(x, t)= & -D_{x}^{\alpha} U(x, t)+2 a U(x, t) D_{x}^{\alpha} U(x, t) \\
& +b D_{t}^{\alpha}\left(D_{x}^{2 \alpha} U(x, t)\right), \quad t>0,0<\alpha \leq 1,
\end{aligned}
$$

where $a$ and $b$ are arbitrary constants. By considering the travelling wave transformation

$$
U(x, t)=U(\xi), \quad \text { with: } \xi=\frac{l x^{\alpha}+\lambda t^{\alpha}}{\Gamma(1+\alpha)},
$$

where $l$ and $\lambda$ are constants, substituting (21) into (20), we can reduce $(20)$ into an ordinary differential equation (ODE):

$$
\begin{aligned}
\lambda \frac{d U(\xi)}{d \xi}= & -l \frac{d U(\xi)}{d \xi}+2 a l U(\xi) \frac{d U(\xi)}{d \xi} \\
& +b \lambda l^{2} \frac{d}{d \xi}\left(\frac{d^{2} U(\xi)}{d \xi^{2}}\right)
\end{aligned}
$$

or

$$
\frac{d^{3} U(\xi)}{d \xi^{3}}=\frac{\lambda+l}{b \lambda l^{2}} \frac{d U(\xi)}{d \xi}-\frac{2 a}{b \lambda l} U(\xi) \frac{d U(\xi)}{d \xi}
$$

Integrating (23) and $\gamma$ being the integration constant, we have

$$
\frac{d^{2} U(\xi)}{d \xi^{2}}=\frac{\lambda+l}{b \lambda l^{2}} U(\xi)-\frac{a}{b \lambda l} U^{2}(\xi)+\gamma
$$

and using (17) and (18), (24) is equivalent to the twodimensional autonomous system

$$
\begin{gathered}
\frac{d X(\xi)}{d \xi}=Y(\xi), \\
\frac{d Y(\xi)}{d \xi}=\frac{\lambda+l}{b \lambda l^{2}} X(\xi)-\frac{a}{b \lambda l} X^{2}(\xi)+\gamma .
\end{gathered}
$$

According to Feng's first integral method, we suppose that $X(\xi)$ and $Y(\xi)$ are nontrivial solutions of $(25)$, and $Q(X, Y)$ is an irreducible polynomial in the complex domain $\mathbb{C}$ such that

$$
Q[X(\xi), Y(\xi)]=\sum_{i=0}^{m} a_{i}(X(\xi)) Y^{i}(\xi)=0
$$

where $a_{i}(X)(i=0,1, \ldots, m)$ are polynomials of $X$ and $a_{m}(X) \neq 0$. Due to the division theorem, there exists a polynomial $g(X)+h(X) Y$ in the complex domain $\mathbb{C}[x, y]$ such that

$$
\begin{aligned}
\frac{d Q}{d \xi} & =\frac{\partial Q}{\partial X} \frac{d X}{d \xi}+\frac{\partial Q}{\partial Y} \frac{d Y}{d \xi} \\
& =(g(X)+h(X) Y(X)) \sum_{i=0}^{m} a_{i}(X) Y^{i}=0 .
\end{aligned}
$$

Suppose that $m=1$; by equating the coefficients of $Y^{i}(i=$ $2,1,0)$ on both sides of $(27)$, we have

$$
\begin{gathered}
\dot{a}_{1}(X)=h(X) a_{1}(X) \\
\dot{a}_{0}(X)=g(X) a_{1}(X)+h(X) a_{0}(X), \\
a_{0}(X) g(X)=a_{1}(X)\left(\frac{\lambda+l}{b \lambda l^{2}} X(\xi)-\frac{a}{b \lambda l} X^{2}(\xi)+\gamma\right) \\
=a_{1}(X)\left(\varepsilon X(\xi)-\beta X^{2}(\xi)+\gamma\right),
\end{gathered}
$$

where

$$
\varepsilon=\frac{\lambda+l}{b \lambda l^{2}}, \quad \beta=\frac{a}{b \lambda l}
$$

since $a_{i}(X)(i=0,1)$ are polynomials, then from (28a) we deduce that $a_{1}(X)$ is constant and $h(X)=0$. For simplicity, we take $a_{1}(X)=1$. Balancing the degrees of $g(X)$ and $a_{0}(X)$, we conclude that

$$
\begin{gathered}
g(X)=g_{m_{0}} X^{m_{0}}+g_{m_{1}} X^{m_{1}} \\
a_{0}(X)=\frac{g_{m_{0}} X^{m_{0}+1}}{m_{0}+1}+\frac{g_{m_{1}} X^{m_{1}+1}}{m_{1}+1}+B_{0}
\end{gathered}
$$

where $B_{0}$ is an arbitrary constant. Substituting $a_{0}(X), a_{1}(X)$, $g(X)$, and $h(X)$ into (28c) and setting all the coefficients of powers of $X$ to be zero, we obtain a system of nonlinear algebraic equations and by solving it, we obtain

$$
\begin{gathered}
m_{0}=-\frac{1}{2}, \quad m_{1}=\frac{1}{2}, \\
B_{0}=0, \quad \gamma=\frac{g_{m_{0}}^{2}}{m_{0}+1}=2 g_{m_{0}}^{2},
\end{gathered}
$$

where

$$
\begin{aligned}
& g_{m_{1}}=\left(-\frac{3}{2} \beta\right)^{1 / 2}, \quad g_{m_{0}}=\frac{3}{8} \varepsilon\left(-\frac{2}{3 \beta}\right)^{1 / 2} \text { or } \\
& g_{m_{1}}=-\left(-\frac{3}{2} \beta\right)^{1 / 2}, \quad g_{m_{0}}=-\frac{3}{8} \varepsilon\left(-\frac{2}{3 \beta}\right)^{1 / 2} .
\end{aligned}
$$

From the above conditions, four solutions can be obtained for the space-time fractional ZKBBM partial differential equation.

Case 1. When $g_{m_{0}}=(3 / 8) \varepsilon(-2 / 3 \beta)^{1 / 2}, g_{m_{1}}=(-(3 / 2) \beta)^{1 / 2}$, $\varepsilon<0$, and $\beta<0$,

$$
a_{0}(X)=2 g_{m_{0}} X^{1 / 2}+\frac{2}{3} g_{m_{1}} X^{3 / 2}
$$

Now from the condition $Q(X, Y)=0$, we obtain

$$
0=a_{0}(X)+a_{1}(X) Y(\xi)
$$

and therefore taking into account that $a_{1}(X)=1$ and from (33) it follows that

$$
Y(\xi)=-\left(2 g_{m_{0}} X^{1 / 2}+\frac{2}{3} g_{m_{1}} X^{3 / 2}\right) .
$$


Combining this first integral $(Y(\xi))$ with the two-dimensional autonomous system of (18), the second order differential equation (24) can be reduced to the following first order differential equation:

$$
\frac{d X(\xi)}{d \xi}=-\left(2 g_{m_{0}} X^{1 / 2}+\frac{2}{3} g_{m_{1}} X^{3 / 2}\right)
$$

We consider the following transformation:

$$
X(\xi)=Z^{2}(\xi),
$$

introduced by Wang [35]. With this transformation (36) can be written as

$$
\frac{d Z(\xi)}{d \xi}=-\left(g_{m_{0}}+\frac{1}{3} g_{m_{1}} Z^{2}\right)
$$

or

$$
\frac{d Z(\xi)}{d \xi}=|\varepsilon|\left(\frac{3}{32|\beta|}\right)^{1 / 2}-\left(\frac{|\beta|}{6}\right)^{1 / 2} Z^{2}
$$

where $\varepsilon=-|\varepsilon|$ and $\beta=-|\beta|$.

By solving (39), the following solutions are obtained:

$$
Z(\xi)=\frac{\sqrt{3|\varepsilon||\beta|}}{2} \frac{\tanh (\sqrt{|\varepsilon| / 8} \xi)}{|\beta|},
$$

or

$$
Z(\xi)=\frac{\sqrt{3|\varepsilon||\beta|}}{2} \frac{\operatorname{coth}(\sqrt{|\varepsilon| / 8} \xi)}{|\beta|},
$$

if we consider the case $l>0$ and $\lambda>0$, and taking into account (29) and (37), the solutions (40) and (41) reduce to

$$
X(\xi)=\frac{6 \sigma b \lambda l}{a} \tanh ^{2}(\sqrt{-\sigma} \xi),
$$

or

$$
X(\xi)=\frac{6 \sigma b \lambda l}{a} \operatorname{coth}^{2}(\sqrt{-\sigma} \xi)
$$

with $\sigma=-|\varepsilon| / 8=(l+\lambda) / 8 b l^{2} \lambda$, where $b<0$ and $a>0$.

Case 2. When $g_{m_{0}}=(3 / 8) \varepsilon(-2 / 3 \beta)^{1 / 2}, g_{m_{1}}=(-(3 / 2) \beta)^{1 / 2}$, $\varepsilon>0$, and $\beta<0$,

$$
a_{0}(X)=2 g_{m_{0}} X^{1 / 2}+\frac{2}{3} g_{m_{1}} X^{3 / 2}
$$

Taking into account that $a_{1}(X)=1$ and from (44) it follows that

$$
Y(\xi)=-\left(2 g_{m_{0}} X^{1 / 2}+\frac{2}{3} g_{m_{1}} X^{3 / 2}\right) .
$$

Combining this first integral $(Y(\xi))$ with the two-dimensional autonomous system given by (18), the second order differential equation (24) can be reduced to the following first order differential equation:

$$
\frac{d X(\xi)}{d \xi}=-\left(2 g_{m_{0}} X^{1 / 2}+\frac{2}{3} g_{m_{1}} X^{3 / 2}\right) .
$$

By using the transformation defined in (37), (46) can be written as

$$
\frac{d Z(\xi)}{d \xi}=-\varepsilon\left(\frac{3}{32|\beta|}\right)^{1 / 2}-\left(\frac{|\beta|}{6}\right)^{1 / 2} Z^{2},
$$

where $\beta=-|\beta|$.

By solving (47), the following solutions are obtained:

$$
Z(\xi)=-\frac{\sqrt{3 \varepsilon|\beta|}}{2} \frac{\tan (\sqrt{\varepsilon / 8} \xi)}{|\beta|},
$$

or

$$
Z(\xi)=-\frac{\sqrt{3 \varepsilon|\beta|}}{2} \frac{\cot (\sqrt{\varepsilon / 8} \xi)}{|\beta|},
$$

if we consider the case $l>0$ and $\lambda>0$, and taking into account (29) and (37), the solutions (48) and (49) reduce to

$$
X(\xi)=-\frac{6 \sigma b \lambda l}{a} \tan ^{2}(\sqrt{\sigma} \xi)
$$

or

$$
X(\xi)=-\frac{6 \sigma b \lambda l}{a} \cot ^{2}(\sqrt{\sigma} \xi)
$$

with $\sigma=\varepsilon / 8=(l+\lambda) / 8 b l^{2} \lambda$, where $b>0$ and $a<0$.

Case 3. When $g_{m_{0}}=-(3 / 8) \varepsilon(-2 / 3 \beta)^{1 / 2}, g_{m_{1}}=-(-(3 /$ 2) $\beta)^{1 / 2}, \varepsilon<0$, and $\beta<0$,

$$
a_{0}(X)=2 g_{m_{0}} X^{1 / 2}+\frac{2}{3} g_{m_{1}} X^{3 / 2} .
$$

Taking into account that $a_{1}(X)=1$ and from (52) it follows that

$$
Y(\xi)=-\left(2 g_{m_{0}} X^{1 / 2}+\frac{2}{3} g_{m_{1}} X^{3 / 2}\right)
$$

Combining this first integral $(Y(\xi))$ with the two-dimensional autonomous system given by (18), the second order differential equation (24) can be reduced to the following first order differential equation:

$$
\frac{d X(\xi)}{d \xi}=-\left(2 g_{m_{0}} X^{1 / 2}+\frac{2}{3} g_{m_{1}} X^{3 / 2}\right)
$$

By using the transformation defined in (37), (54) can be written as

$$
\frac{d Z(\xi)}{d \xi}=-|\varepsilon|\left(\frac{3}{32|\beta|}\right)^{1 / 2}+\left(\frac{|\beta|}{6}\right)^{1 / 2} Z^{2},
$$

where $\varepsilon=-|\varepsilon|$ and $\beta=-|\beta|$.

By solving (55), the following solutions are obtained:

$$
Z(\xi)=-\frac{\sqrt{3|\varepsilon||\beta|}}{2} \frac{\tanh (\sqrt{|\varepsilon| / 8} \xi)}{|\beta|},
$$


or

$$
Z(\xi)=-\frac{\sqrt{3|\varepsilon||\beta|}}{2} \frac{\operatorname{coth}(\sqrt{|\varepsilon| / 8} \xi)}{|\beta|},
$$

if we consider the case $l>0$ and $\lambda>0$, and taking into account (29) and (37), the solutions (56) and (57) reduce to

$$
X(\xi)=\frac{6 \sigma b \lambda l}{a} \tanh ^{2}(\sqrt{-\sigma} \xi),
$$

or

$$
X(\xi)=\frac{6 \sigma b \lambda l}{a} \operatorname{coth}^{2}(\sqrt{-\sigma} \xi)
$$

with $\sigma=-|\varepsilon| / 8=(l+\lambda) / 8 b l^{2} \lambda$, where $b<0$ and $a>0$.

Case 4. When $g_{m_{0}}=-(3 / 8) \varepsilon(-2 / 3 \beta)^{1 / 2}, g_{m_{1}}=-(-(3 /$ 2) $\beta)^{1 / 2}, \varepsilon>0$, and $\beta<0$,

$$
a_{0}(X)=2 g_{m_{0}} X^{1 / 2}+\frac{2}{3} g_{m_{1}} X^{3 / 2}
$$

Taking into account that $a_{1}(X)=1$ and from (60) it follows that

$$
Y(\xi)=-\left(2 g_{m_{0}} X^{1 / 2}+\frac{2}{3} g_{m_{1}} X^{3 / 2}\right)
$$

Combining this first integral $(Y(\xi))$ with the two-dimensional autonomous system given by (18), the second order differential equation (24) can be reduced to the following first order differential equation:

$$
\frac{d X(\xi)}{d \xi}=-\left(2 g_{m_{0}} X^{1 / 2}+\frac{2}{3} g_{m_{1}} X^{3 / 2}\right)
$$

By using the transformation defined in (37), (62) can be written as

$$
\frac{d Z(\xi)}{d \xi}=+\varepsilon\left(\frac{3}{32|\beta|}\right)^{1 / 2}+\left(\frac{|\beta|}{6}\right)^{1 / 2} Z^{2}
$$

where $\beta=-|\beta|$.

By solving (63), the following solutions are obtained:

$$
Z(\xi)=\frac{\sqrt{3 \varepsilon|\beta|}}{2} \frac{\tan (\sqrt{\varepsilon / 8} \xi)}{|\beta|},
$$

or

$$
Z(\xi)=\frac{\sqrt{3 \varepsilon|\beta|}}{2} \frac{\cot (\sqrt{\varepsilon / 8} \xi)}{|\beta|},
$$

if we consider the case $l>0$ and $\lambda>0$, and taking into account (29) and (37), the solutions (64) and (65) reduce to

$$
X(\xi)=-\frac{6 \sigma b \lambda l}{a} \tan ^{2}(\sqrt{\sigma} \xi)
$$

or

$$
X(\xi)=-\frac{6 \sigma b \lambda l}{a} \cot ^{2}(\sqrt{\sigma} \xi)
$$

with $\sigma=\varepsilon / 8=(l+\lambda) / 8 b l^{2} \lambda$, where $b>0$ and $a<0$.

For the nonlinear space-time fractional ZKBBM equation, we have successfully recovered the previously known solutions, (42), (43), (48), (49), (58), (59), (66), and (67), that have been found in [29]; furthermore, Feng's first integral method has evaluated the constant $\sigma$ which has not been evaluated in this previous work.

Example 2. Next we consider the nonlinear fractional generalized Fisher equation (11):

$$
\begin{array}{r}
D_{t}^{\alpha} U(x, t)=D_{x}^{2 \alpha} U(x, t)+U(x, t)\left(1-U^{6}(x, t)\right), \\
t>0, \quad 0<\alpha \leq 1 .
\end{array}
$$

By considering the following transformation,

$$
U^{3}(x, t)=y(x, t),
$$

introduced by Wang [35], (68) can be written as

$$
\begin{aligned}
y(x, t) & D_{t}^{\alpha} y(x, t) \\
= & y(x, t) D_{x}^{2 \alpha} y(x, t)-\frac{2}{3}\left(D_{x}^{\alpha} y(x, t)\right)^{2} \\
& +3 y^{2}(x, t)\left(1-y^{2}(x, t)\right), \quad t>0,0<\alpha \leq 1 .
\end{aligned}
$$

By considering the travelling wave transformation,

$$
y(x, t)=y(\xi), \quad \text { with: } \xi=\frac{l x^{\alpha}+\lambda t^{\alpha}}{\Gamma(1+\alpha)},
$$

where $l$ and $\lambda$ are constants, (70) can be reduced to the following nonlinear ordinary differential equation:

$$
\begin{aligned}
\lambda y(\xi) \frac{d y(\xi)}{d \xi}= & l^{2} y(\xi) \frac{d^{2} y(\xi)}{d \xi^{2}}-\frac{2}{3} l^{2}\left(\frac{d y(\xi)}{d \xi}\right)^{2} \\
& +3 y^{2}(\xi)\left(1-y^{2}(\xi)\right), \quad 0<\alpha \leq 1 .
\end{aligned}
$$

We assume that (72) has a solution in the form $y(\xi)=X(\xi)$, and using (18), (72) is equivalent to the two-dimensional autonomous system:

$$
\begin{gathered}
\frac{d X(\xi)}{d \xi}=Y(\xi), \\
l^{2} X(\xi) \frac{d Y(\xi)}{d \xi}=\lambda X(\xi) Y(\xi)+\frac{2}{3} l^{2}(Y(\xi))^{2} \\
-3 X^{2}(\xi)\left(1-X^{2}(\xi)\right), \quad 0<\alpha \leq 1 .
\end{gathered}
$$

According to Feng's first integral method, we suppose that $X(\xi)$ and $Y(\xi)$ are nontrivial solutions of $(73)$, and $Q(X, Y)$ 
is an irreducible polynomial in the complex domain $\mathbb{C}$ such that

$$
Q[X(\xi), Y(\xi)]=\sum_{i=0}^{m} a_{i}(X(\xi)) Y^{i}(\xi)=0,
$$

where $a_{i}(X)(i=0,1, \ldots, m)$ are polynomials of $X$ and $a_{m}(X) \neq 0$. Due to the division theorem, there exists a polynomial $g(X)+h(X) Y$ in the complex domain $\mathbb{C}[X, Y]$ such that

$$
\begin{aligned}
\frac{d Q}{d \xi} & =\frac{\partial Q}{\partial X} \frac{d X}{d \xi}+\frac{\partial Q}{\partial Y} \frac{d Y}{d \xi} \\
& =(g(X)+h(X) Y(X)) \sum_{i=0}^{m} a_{i}(X) Y^{i}=0 .
\end{aligned}
$$

Suppose that $m=1$; by equating the coefficients of $Y^{i}(i=$ $2,1,0)$ on both sides of $(75)$, we have

$$
\begin{gathered}
\dot{a}_{1}(X) X(\xi)=a_{1}(X)\left[h(X) X(\xi)-\frac{2}{3}\right], \\
\dot{a}_{0}(X)+a_{1}(X) \frac{\lambda}{l^{2}}=a_{1}(X) g(X)+a_{0}(X) h(X), \\
a_{0}(X) g(X) X(\xi) l^{2}=-3 a_{1} X^{2}(\xi)\left(1-X^{2}(\xi)\right) .
\end{gathered}
$$

Since $a_{i}(X)(i=0,1)$ are polynomials, then from (76a) we deduce that $a_{1}(X)$ is constant and $h(X)=2 / 3 X$. For simplicity, we take $a_{1}(X)=1$. Balancing the degrees of $g(X)$ and $a_{0}(X)$, we conclude that

$$
\begin{gathered}
g(X)=A_{0}+B_{0} X \\
a_{0}(X)=\mathscr{A}_{0}+\mathscr{B}_{0} X+\frac{\mathscr{C}_{0} X^{2}}{2} .
\end{gathered}
$$

Substituting $a_{0}(X), a_{1}(X), g(X)$, and $h(X)$ into (76c) and setting all the coefficients of powers of $X$ to be zero, we obtain a system of nonlinear algebraic equations and by solving it, we obtain

$$
\begin{gathered}
\mathscr{A}_{0}=0, \quad \mathscr{B}_{0}=3\left(A_{0}-\frac{\lambda}{l^{2}}\right), \\
\mathscr{C}_{0}=\frac{3}{2} B_{0}, \quad \lambda=\frac{5}{4} A_{0} l^{2}, \\
A_{0}^{2}=\frac{4}{l^{2}}, \quad B_{0}^{2}=\frac{4}{l^{2}} ;
\end{gathered}
$$

therefore we obtain the following set of solutions:

$$
A_{0}= \pm \frac{2}{l}, \quad B_{0}= \pm \frac{2}{l}
$$

From the above conditions, four solutions can be obtained.

Case 1. When $A_{0}=2 / l$ and $B_{0}=2 / l$,

$$
a_{0}(X)=-\frac{3}{2 l} X+\frac{3}{2 l} X^{2} .
$$

Now from the condition $Q(X, Y)=0$ we obtain

$$
0=a_{0}(X)+a_{1}(X) Y(\xi),
$$

and therefore taking into account that $a_{1}(X)=1$ and from (81) it follows that

$$
Y(\xi)=\frac{3}{2 l} X-\frac{3}{2 l} X^{2}
$$

If we consider the definition given in (18) one finally obtains

$$
\frac{d X(\xi)}{d \xi}=\frac{3}{2 l} X-\frac{3}{2 l} X^{2}
$$

By solving the above equation the solution to (72) is obtained:

$$
X(\xi)=\frac{1}{1+c_{1} e^{-3 \xi / 2 l}} ;
$$

if we consider the case $c_{1}=1$, the solution (84) reduces to

$$
X(\xi)=\frac{1}{2}\left(1+\tanh \left(\frac{3 \xi}{4 l}\right)\right),
$$

with $\lambda=5 l / 2$. Taking into account (69), the solution to the Fisher equation (68) is given by

$$
U(x, t)=\left(\frac{1}{2}\left(1+\tanh \left(\frac{3 \xi}{4 l}\right)\right)\right)^{1 / 3},
$$

with

$$
\xi=\frac{l x^{\alpha}+\lambda t^{\alpha}}{\Gamma(1+\alpha)} .
$$

Case 2. When $A_{0}=-2 / l$ and $B_{0}=2 / l$,

$$
a_{0}(X)=\frac{3}{2 l} X+\frac{3}{2 l} X^{2}
$$

Now from the condition $Q(X, Y)=0$ we obtain

$$
0=a_{0}(X)+a_{1}(X) Y(\xi)
$$

and therefore taking into account that $a_{1}(X)=1$ and from (88), it follows that

$$
Y(\xi)=-\left(\frac{3}{2 l} X+\frac{3}{2 l} X^{2}\right)
$$

If we consider the definition given in (18), one finally obtains

$$
\frac{d X(\xi)}{d \xi}=-\left(\frac{3}{2 l} X+\frac{3}{2 l} X^{2}\right)
$$

By solving the above equation, the solution to (72) is obtained:

$$
X(\xi)=\frac{1}{c_{1} e^{3 \xi / 2 l}-1} ;
$$

if we consider the case $c_{1}=1$, the solution (92) reduces to

$$
X(\xi)=-\frac{1}{2}\left(1-\operatorname{coth}\left(\frac{3 \xi}{4 l}\right)\right),
$$


with $\lambda=-5 l / 2$. Taking into account (69), the solution to the Fisher equation (68) is given by

$$
U(x, t)=\left(-\frac{1}{2}\left(1-\operatorname{coth}\left(\frac{3 \xi}{4 l}\right)\right)\right)^{1 / 3},
$$

with

$$
\xi=\frac{l x^{\alpha}+\lambda t^{\alpha}}{\Gamma(1+\alpha)} .
$$

Case 3. When $A_{0}=2 / l$ and $B_{0}=-2 / l$,

$$
a_{0}(X)=-\left(\frac{3}{2 l} X+\frac{3}{2 l} X^{2}\right)
$$

Now from the condition $Q(X, Y)=0$, we obtain

$$
0=a_{0}(X)+a_{1}(X) Y(\xi)
$$

and therefore taking into account that $a_{1}(X)=1$ and from (96), it follows that

$$
Y(\xi)=\left(\frac{3}{2 l} X+\frac{3}{2 l} X^{2}\right) .
$$

If we consider the definition given in (18), one finally obtains

$$
\frac{d X(\xi)}{d \xi}=\left(\frac{3}{2 l} X+\frac{3}{2 l} X^{2}\right) .
$$

By solving the above equation, the solution to (72) is obtained:

$$
X(\xi)=\frac{1}{c_{1} e^{-3 \xi / 2 l}-1} ;
$$

if we consider the case $c_{1}=1$, the solution (100) reduces to

$$
X(\xi)=-\frac{1}{2}\left(1+\operatorname{coth}\left(\frac{3 \xi}{4 l}\right)\right),
$$

with $\lambda=5 l / 2$. Taking into account (69), the solution to the Fisher equation (68) is given by

$$
U(x, t)=\left(-\frac{1}{2}\left(1+\operatorname{coth}\left(\frac{3 \xi}{4 l}\right)\right)\right)^{1 / 3},
$$

with

$$
\xi=\frac{l x^{\alpha}+\lambda t^{\alpha}}{\Gamma(1+\alpha)} .
$$

Case 4. When $A_{0}=-2 / l$ and $B_{0}=-2 / l$,

$$
a_{0}(X)=\frac{3}{2 l} X-\frac{3}{2 l} X^{2}
$$

Now from the condition $Q(X, Y)=0$, we obtain

$$
0=a_{0}(X)+a_{1}(X) Y(\xi)
$$

and therefore taking into account that $a_{1}(X)=1$ and from (104), it follows that

$$
Y(\xi)=-\left(\frac{3}{2 l} X-\frac{3}{2 l} X^{2}\right)
$$

If we consider the definition given in (18), one finally obtains

$$
\frac{d X(\xi)}{d \xi}=-\left(\frac{3}{2 l} X-\frac{3}{2 l} X^{2}\right)
$$

By solving the above equation, the solution to (72) is obtained:

$$
X(\xi)=\frac{1}{c_{1} e^{3 \xi / 2 l}+1}
$$

if we consider the case $c_{1}=1$, the solution (108) reduces to

$$
X(\xi)=\frac{1}{2}\left(1-\tanh \left(\frac{3 \xi}{4 l}\right)\right),
$$

with $\lambda=-5 l / 2$. Taking into account (69), the solution to the Fisher equation (68) is given by

$$
U(x, t)=\left(\frac{1}{2}\left(1-\tanh \left(\frac{3 \xi}{4 l}\right)\right)\right)^{1 / 3}
$$

with

$$
\xi=\frac{l x^{\alpha}+\lambda t^{\alpha}}{\Gamma(1+\alpha)}
$$

For the nonlinear space-time generalized fractional Fisher equation, we have successfully recovered the previously known solution (110) that has been found in [31], but to the best of our knowledge the solutions (86), (94), and (102) have not been obtained previously in the literature.

\section{Conclusions}

Feng's first integral method was applied successfully to obtain new exact analytical solutions of the nonlinear space-time fractional ZKBBM equation and the nonlinear space-time fractional generalized Fisher equation. These solutions can be very useful as a starting point of comparison when some approximate methods are applied to these nonlinear space-time fractional equations. The performance of Feng's first integral method is reliable and effective to obtain new solutions. This method has more advantages: it is direct and concise. Thus, the proposed method can be extended to solve many systems of nonlinear fractional partial differential equations in mathematical and physical sciences.

\section{Conflict of Interests}

The authors declare that there is no conflict of interests regarding the publication of this paper.

\section{Acknowledgment}

The authors gratefully acknowledge the Universidad Autónoma de la Ciudad de Mexico for supporting and facilitating this research work. 


\section{References}

[1] K. S. Miller and B. Ross, An Introduction to the Fractional Calculus and Fractional Differential Equations, John Wiley \& Sons, New York, NY, USA, 1993.

[2] A. A. Kilbas, H. M. Srivastava, and J. J. Trujillo, Theory and Applications of Fractional Differential Equations, vol. 204 of North-Holland Mathematics Studies, Elsevier Science, Amsterdam, The Netherlands, 2006.

[3] I. Podlubny, Fractional Differential Equations, vol. 198 of Mathematics in Science and Engineering, Academic Press, San Diego, Calif, USA, 1999.

[4] A. M. El-Sayed, S. Z. Rida, and A. A. Arafa, "Exact solutions of fractional-order biological population model," Communications in Theoretical Physics, vol. 52, no. 6, pp. 992-996, 2009.

[5] M. Safari, D. D. Ganji, and M. Moslemi, "Application of He's variational iteration method and Adomian's decomposition method to the fractional KdV-Burgers-KURamoto equation," Computers \& Mathematics with Applications, vol. 58, no. 11-12, pp. 2091-2097, 2009.

[6] M. Inc, "The approximate and exact solutions of the spaceand time-fractional Burgers equations with initial conditions by variational iteration method," Journal of Mathematical Analysis and Applications, vol. 345, no. 1, pp. 476-484, 2008.

[7] G.-c. Wu and E. W. Lee, "Fractional variational iteration method and its application," Physics Letters, A, vol. 374, no. 25, pp. 25062509, 2010.

[8] F. Fouladi, E. Hosseinzadeh, A. Barari, and G. Domairry, "Highly nonlinear temperature-dependent fin analysis by variational iteration method," Heat Transfer Research, vol. 41, no. 2, pp. 155-165, 2010.

[9] L. Song and H. Zhang, "Solving the fractional BBM-Burgers equation using the homotopy analysis method," Chaos, Solitons and Fractals, vol. 40, no. 4, pp. 1616-1622, 2009.

[10] S. Abbasbandy and A. Shirzadi, "Homotopy analysis method for multiple solutions of the fractional Sturm-Liouville problems," Numerical Algorithms, vol. 54, no. 4, pp. 521-532, 2010.

[11] H. Bararnia, G. Domairry, M. Gorji, and A. Rezania, "An approximation of the analytic solution of some nonlinear heat transfer in fin and 3D diffusion equations using HAM," Numerical Methods for Partial Differential Equations, vol. 26, no. 1, pp. 1-13, 2010.

[12] M. M. Rashidi, G. Domairry, A. DoostHosseini, and S. Dinarvand, "Explicit approximate solution of the coupled KdV equations by using the homotopy analysis method," International Journal of Mathematical Analysis, vol. 2, no. 9-12, pp. 581-589, 2008.

[13] Z. Z. Ganji, D. D. Ganji, A. D. Ganji, and M. Rostamian, "Analytical solution of time-fractional Navier-Stokes equation in polar coordinate by homotopy perturbation method," Numerical Methods for Partial Differential Equations, vol. 26, no. 1, pp. 117-124, 2010.

[14] K. A. Gepreel, "The homotopy perturbation method applied to the nonlinear fractional Kolmogorov-Petrovskii-PISkunov equations," Applied Mathematics Letters, vol. 24, no. 8, pp. 14281434, 2011.

[15] P. K. Gupta and M. Singh, "Homotopy perturbation method for fractional Fornberg-Whitham equation," Computers \& Mathematics with Applications, vol. 61, no. 2, pp. 250-254, 2011.

[16] J. Guy, "Lagrange characteristic method for solving a class of nonlinear partial differential equations of fractional order," Applied Mathematics Letters, vol. 19, no. 9, pp. 873-880, 2006.
[17] S. Zhang and H.-Q. Zhang, "Fractional sub-equation method and its applications to nonlinear fractional PDEs," Physics Letters A, vol. 375, no. 7, pp. 1069-1073, 2011.

[18] G. Jumarie, "Modified Riemann-Liouville derivative and fractional Taylor series of nondifferentiable functions further results," Computers \& Mathematics with Applications, vol. 51, no. 9-10, pp. 1367-1376, 2006.

[19] Z. Feng, "The first-integral method to study the BurgersKorteweg-de Vries equation," Journal of Physics A: Mathematical and General, vol. 35, no. 2, pp. 343-349, 2002.

[20] Z. Feng and R. Knobel, "Traveling waves to a BurgersKorteweg-de Vries-type equation with higher-order nonlinearities," Journal of Mathematical Analysis and Applications, vol. 328, no. 2, pp. 1435-1450, 2007.

[21] Z. Feng, "Traveling wave behavior for a generalized Fisher equation," Chaos, Solitons and Fractals, vol. 38, no. 2, pp. 481488, 2008.

[22] K. R. Raslan, "The first integral method for solving some important nonlinear partial differential equations," Nonlinear Dynamics, vol. 53, no. 4, pp. 281-286, 2008.

[23] B. Lu, H. Zhang, and F. Xie, "Travelling wave solutions of nonlinear partial equations by using the first integral method," Applied Mathematics and Computation, vol. 216, no. 4, pp. 13291336,2010

[24] N. Taghizadeh, M. Mirzazadeh, and F. Farahrooz, "Exact solutions of the nonlinear Schrödinger equation by the first integral method," Journal of Mathematical Analysis and Applications, vol. 374, no. 2, pp. 549-553, 2011.

[25] B. Lu, "The first integral method for some time fractional differential equations," Journal of Mathematical Analysis and Applications, vol. 395, no. 2, pp. 684-693, 2012.

[26] M. Eslami, B. Fathi Vajargah, M. Mirzazadeh, and A. Biswas, "Application of first integral method to fractional partial differential equations," Indian Journal of Physics, vol. 88, no. 2, pp. 177-184, 2014.

[27] T. B. Benjamin, J. L. Bona, and J. J. Mahony, "Model equations for long waves in nonlinear dispersive systems," Philosophical Transactions of the Royal Society of London A, vol. 272, no. 1220, pp. 47-78, 1972.

[28] A.-M. Wazwaz, "The extended tanh method for new compact and noncompact solutions for the KP-BBM and the ZK-BBM equations," Chaos, Solitons and Fractals, vol. 38, no. 5, pp. 1505$1516,2008$.

[29] J. Alzaidy, "Fractional sub-equation method and its applications to the space-time fractional differential equations in mathematical physics," British Journal of Mathematics \& Computer Science, vol. 3, no. 2, pp. 153-163, 2013.

[30] R. A. Fisher, "The wave of advance of advantageous genes," Annals of Eugenics, vol. 7, no. 4, pp. 355-369, 1937.

[31] D. Ağirseven and T. Özis, "An analytical study for Fisher type equations by using homotopy perturbation method," Computers \& Mathematics with Applications, vol. 60, no. 3, pp. 602-609, 2010.

[32] J. D. Murray, Mathematical biology, vol. 19 of Biomathematics Texts, Springer, Berlin, Germany, 2nd edition, 1993.

[33] A. G. Nikitin and T. A. Barannyk, "Solitary wave and other solutions for nonlinear heat equations," Central European Journal of Mathematics, vol. 2, no. 5, pp. 840-858, 2004.

[34] N. Bourbaki, Elements of Mathematics. Commutative Algebra, Hermann, Addison-Wesley, Paris, France, 1972.

[35] X. Y. Wang, "Exact and explicit solitary wave solutions for the generalised Fisher equation," Physics Letters, A, vol. 131, no. 4-5, pp. $277-279,1988$. 


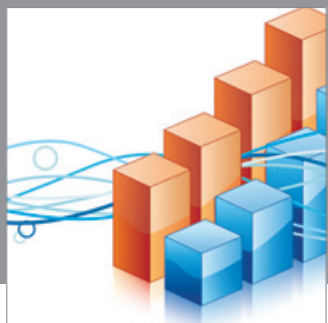

Advances in

Operations Research

mansans

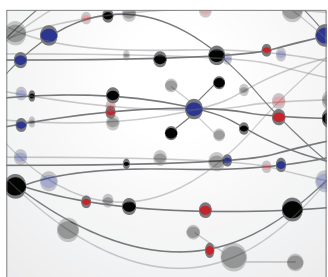

The Scientific World Journal
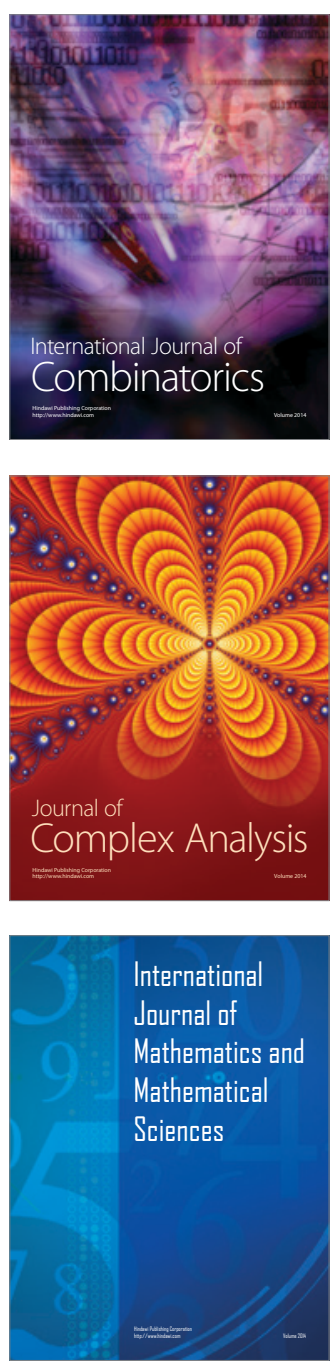
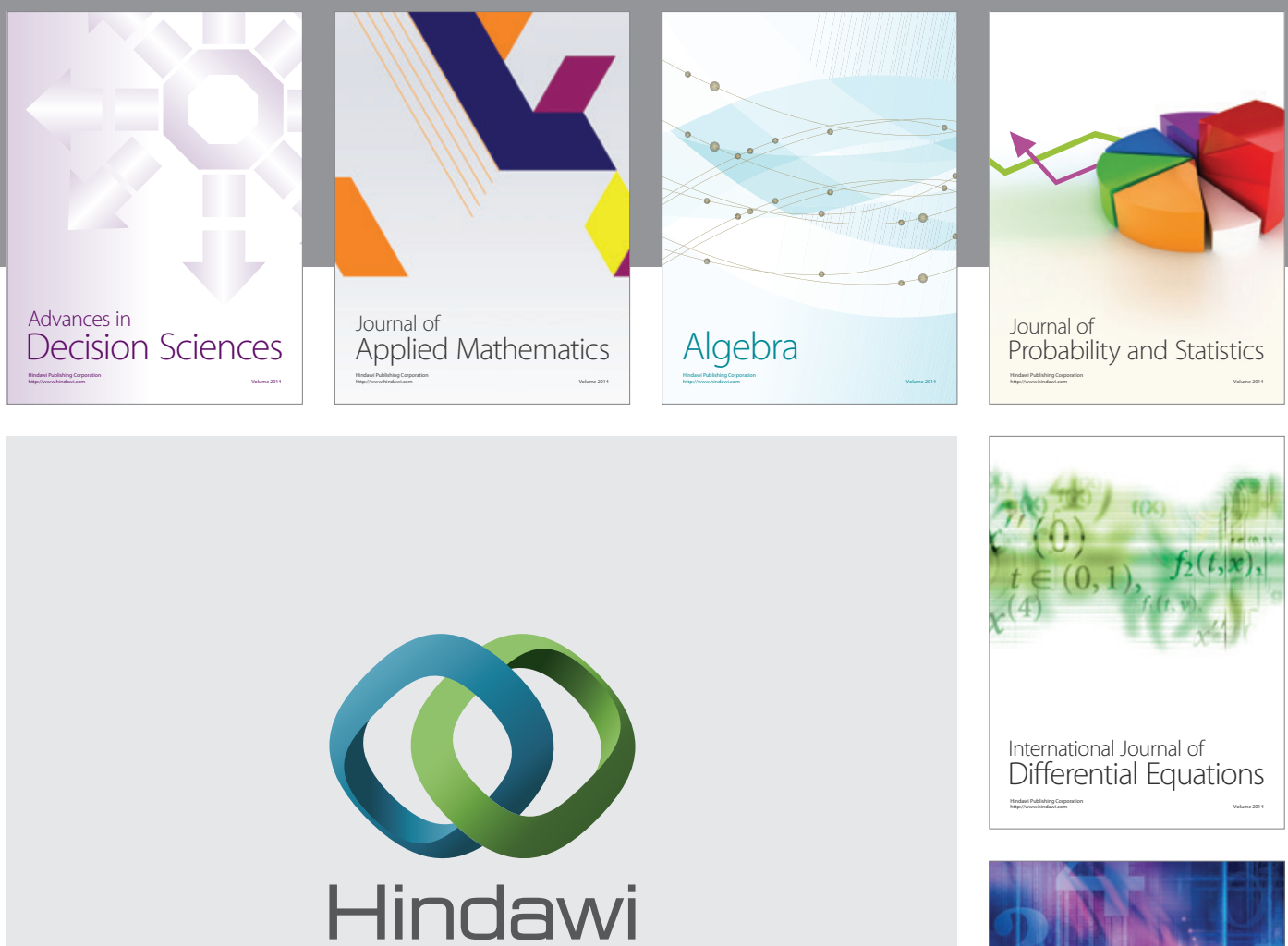

Submit your manuscripts at http://www.hindawi.com
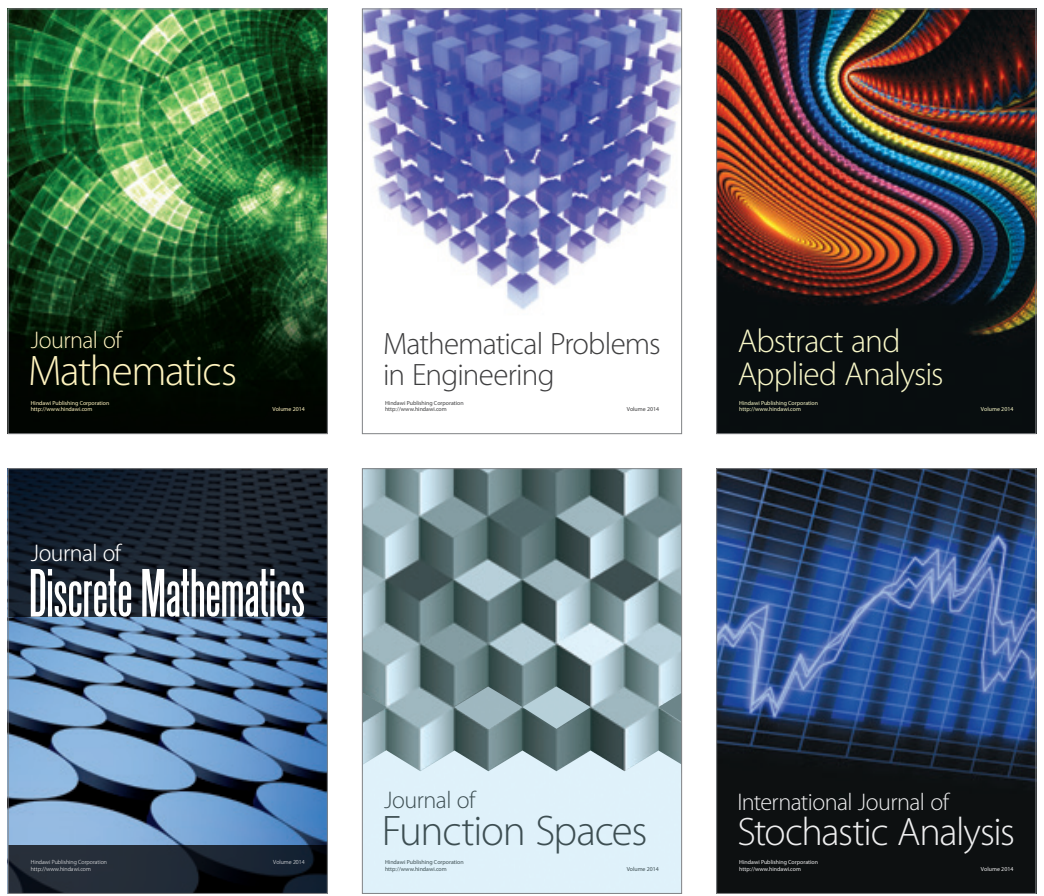

Journal of

Function Spaces

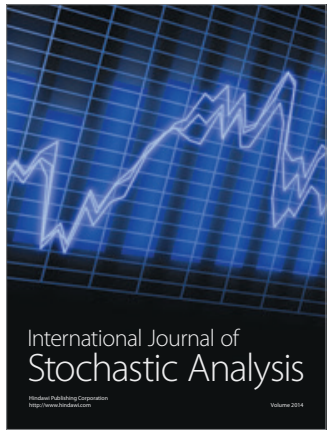

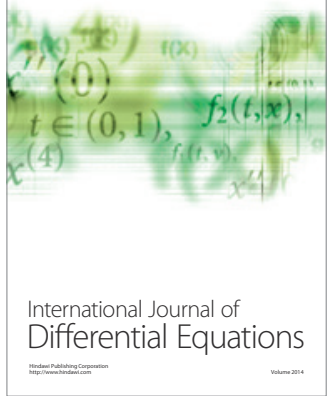
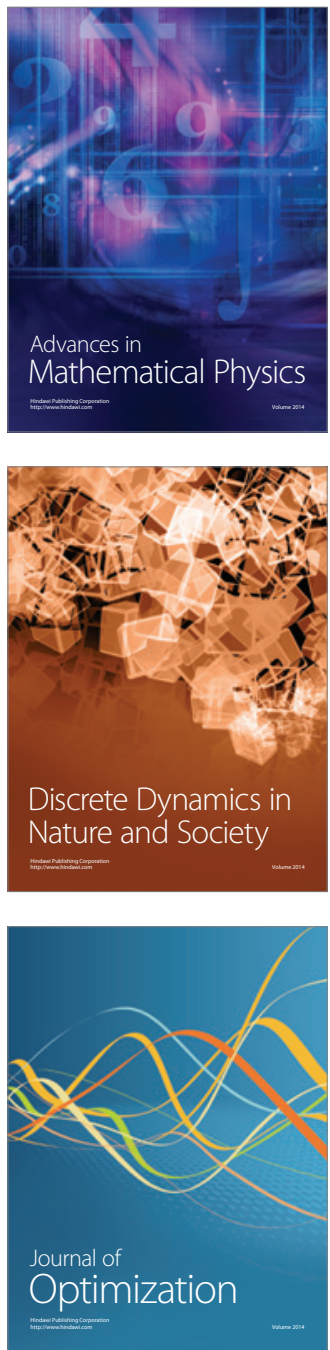